\title{
Adesione batterica e fungina a materiali protesici del cavo orale
}

\author{
Fabrizio Cavallini', Simone Cagnacci', Elisabetta Maioli', Lionello Lagorio², \\ Damiano Gibelli', Simona Roveta', Anna Marchese', Eugenio A. Debbia' \\ 'Sezione di Microbiologia, DISCAT, \\ ${ }^{2}$ Sezione di Odontostomatologia, DISTBIMO Università di Genova
}

Parole chiave: materiali protesici, cavo orale adesività

\section{SUMMARY}

Introduction. The microbial adhesion to dental material was studied employing a acrylic resin and representative microrganisms.

Method. Bacterial suspensions (about $10^{8} \mathrm{CFU} / \mathrm{ml}$ ) were mixed to dental material with different surface finishes. Bacterial counts were carried out before and after I hour at $37^{\circ} \mathrm{C}$. A tube without dental material was used as control. The same experiments were also performed at the presence of saliva.

Results. Candida albicans demonstrated to adhere to free energy material at an incidence $64 \%$ while Staphylococcus aureus and Streptococcus oralis showed more adhesive properties for roughness material (34 and $52 \%$ respectively). The presence of saliva influenced the adhesiveness to all materials with the exception of $S$. aureus that increased from 20 to $29 \%$ the number of cells on the surface of free energy material.

Conclusion. The surface finishes appeared did not significantly influence the adhesion of microrganisms to this material.

\section{INTRODUZIONE}

L'inserimento di un dispositivo protesico rimovibile, parziale o totale, nella cavità orale ne modifica sensibilmente la flora batterica sia in senso quantitativo che qualitativo. L'alterazione e l'accumulo di placca batterica possono comportare lo sviluppo di condizioni patologiche, come la carie e la malattia parodontale sugli elementi residui in portatori protesi parziali $(6,17)$ e la stomatite da protesi in portatori di protesi completa $(3,4)$. L'entità e la natura delle modificazioni della flora orale dipendono sostanzialmente dalle caratteristiche strutturali della protesi (dimensioni, forma, materiali costituenti), mentre il verificarsi di situazioni patologiche dipende in larga misura dal mantenimento di un'adeguata igiene delle protesi e degli elementi dentari residui da parte del paziente $(5,18)$.

Uno degli aspetti fondamentali che occorre considerare è la capacità di adesione da parte dei microrganismi della cavità orale sulla superficie delle protesi, che rappresenta il presupposto indispensabile l'eventuale sviluppo di condizioni patologiche.

Due principali fattori influenzano l'adesione batterica e micotica ai materiali di uso protesico: l'energia superficiale libera e la ruvidità superficiale (11). Dal momento che la ruvidità superficiale sembra giocare un ruolo preminente (10), i materiali dentari utilizzati per la costruzione di protesi vengono sottoposti a diversi trattamenti di levigatura. Ogni materiale necessita della propria modalità di trattamento al fine di ottenere una superfi- cie quanto più possibile regolare (1) e ogni strumento rotante produce delle caratteristiche di superficie peculiari sul materiale (14).

Molti studi sperimentali sono stati condotti per valutare le capacità adesive di vari ceppi di microrganismi ai materiali per basi protesiche.

È stato rilevato che le procedure di lucidatura non condizionano sensibilmente l'adesione di Streptococcus oralis e Actinomyces viscosus su quattro tipi di resina acrilica a freddo o a caldo (2), tuttavia è stato altresì evidenziato che il tipo di resina acrilica e la sua ruvidità influenzano significativamente la precoce formazione di un biofilm aderente da parte di S. oralis (7). Mentre A. viscosus aderisce preferibilmente a superfici ruvide, $S$. oralis sembra prediligere le superfici lucide. Infatti l'adesione di queste due specie batteriche così come quella di Candida albicans avviene in maniera preferenziale su una superficie in funzione del tipo di ruvidità della superficie stessa e delle differenti dimensioni dei microrganismi (15).

Una superficie ruvida favorisce l'adesione da parte di C. albicans tanto a materiali acrilici quanto, in maniera ancora più consistente, a materiali morbidi per ribasamento (13). Per entrambi i tipi di materiale, l'aderenza fungina diminuisce in presenza di pellicola salivare. Analoghe osservazioni hanno svolto Verran et al. (16), che riferiscono un sensibile aumento di aderenza di $C$. albicans su resina acrilica e silicone per ribasamento morbido quando non levigati. Proprio i siliconi per ribasamento morbido presentano le 
caratteristiche di rifinitura e lucidabilità meno favorevoli $(13,14)$. In realtà, come sottolineano Radford et al. (12), il fenomeno dell'adesione di C. albicans alle basi protesiche risulta verosimilmente molto complesso e articolato. Un fattore da considerare, ad esempio, è l'effetto di competizione da parte di altri batteri commensali del cavo orale sull'adesione sulla superficie acrilica protesica. Nair e Samaranayake (8), ad esempio, hanno evidenziato come la pre-esposizione a Streptococcus sanguis e Porphyromonas gengivalis riduca sensibilmente le capacità adesive di $C$. albicans a campioni di resina acrilica.

Scopo di questo lavoro è svolgere una valutazione comparativa del grado di adesione di tre microorganismi su campioni di tre materiali protesici levigati e non levigati (ruvidi).

\section{MATERIALI E METODI}

I microorganismi utilizzati in questo studio sono stati isolati da materiale patologico, nella collezione sono stati inclusi Staphylococcus aureus, S. oralis, C. albicans.

Sono state allestite sospensioni delle varie specie batteriche in soluzione PBS alla concentrazione di almeno $10^{8} \mathrm{CFU} / \mathrm{ml}$, alle varie sospensioni sono stati aggiunti i campioni sferici di materiale protesico di cui si voleva saggiare l'adesività. La stima delle cellule batteriche in sospensione è stata fatta prima e dopo un'ora di incubazione a $37^{\circ} \mathrm{C}$. la differenza tra il numero iniziale e quello finale ha dato la quantità di cellule adese al materiale. Una sospensione microbica priva di materiale protesico è stata utilizzata come controllo.

Per gli esperimenti effettuati in presenza di saliva, è stato allestito un campione di saliva nel seguente modo: la saliva è stata raccolta da un soggetto che non aveva preso farmaci nei tre mesi precedenti lo svolgimento degli esperimenti; inoltre erano assenti processi cariosi e malattie parodontali.

La saliva raccolta è stata centrifugata a $5000^{*} \mathrm{~g}$ a $4^{\circ} \mathrm{C}$ e in seguito conservata in frigorifero a $4^{\circ} \mathrm{C}$.

\section{Preparazione del materiale campione}

La realizzazione delle pastiglie-campione è avvenuta seguendo fondamentali passaggi di laboratorio: inizialmente tramite la modellazione in cera che ha permesso di darne forma e grandezza desiderate ed in seguito con la costruzione di stampi per la duplicazione delle pastiglie-campione. Date le differenti caratteristiche del materiale da utilizzare, si è proceduto alla realizzazione con due diverse tecniche: polimerizzazione a caldo, polimerizzazione a freddo.

La tecnica della polimerizzazione a caldo prevede la messa in muffola delle pastiglie-campione in cera con la realizzazione del relativo stampo e controstampo in gesso duro. Ottenuto l'indurimento del gesso si è proceduto alla rimozione della cera attraverso un lavaggio a temperatura di ebollizione. Successivamente è stato applicato con un pennello del liquido isolante sulle superfici in gesso e una volta raffreddato il tutto è stato preparato l'impasto in resina per basi da dentiera (paladon) con un rapporto polvere/liquido di $10 \mathrm{~g}: 4 \mathrm{ml}$ o multipli. Ottenuto l'impasto di consistenza morbida-elastica e non filamentosa si è proceduto al riempimento degli spazi lasciati vuoti dalle pastiglie in cera; con una pressione di $2 \mathrm{~kg}$ x 1000 bar si metteva in staffa, permettendo così di mantenere la pressione esercitata evitando la formazione di porosità. Seguiva poi la fase della cottura che avveniva in due tempi: dapprima immergendo la muffola in acqua fino a raggiungere $70^{\circ} \mathrm{C}$ mantenendo la temperatura costante per circa mezz'ora e poi attraverso un lento innalzamento della temperatura si portava ad ebollizione per almeno $30 \mathrm{~min}$. Terminato il ciclo di polimerizzazione si smuffolavano le pastiglie-campione e si passava alla rifinitura con frese al carbonio-tungsteno a taglio incrociato e con carta smerigliata. Delle sei pastiglie-campione ottenute tre venivano lasciate con superficie ruvida, tre venivano trattate con gommino, feltrino e pasta per lucidare ottenendo così una superficie liscia e lucida.

La tecnica della polimerizzazione a freddo (autopolimerizzazione) è stata utilizzata per la realizzazione delle rimanenti pastiglie-campione. Utilizzando le pastiglie-campione in cera, precedentemente modellate, si costruiva lo stampo in silicone che una volta indurito veniva deterso con acqua calda prestando attenzione a non alterarne la forma. Realizzato lo stampo si miscelavano polvere/liquido sempre in rapporto $2 / 1$ per le resine al fine di ottenere l'impasto cremoso con cui si sarebbero riempite le formine precedentemente preparate. Il tutto veniva posto in una pentola a pressione a 1,5 ATM per circa 5-10 minuti a una temperatura di $40^{\circ}-45^{\circ} \mathrm{C}$. A polimerizzazione avvenuta il materiale risultava più compatto, privo di bolle d'aria. Per il silicone si miscelavano i due componenti ossia la pasta base ed il catalizzatore ripetendo i passaggi sopra descritti. Al termine della completa polimerizzazione si rifinivano le pastiglie-campione in resina autopolimerizzante con frese al tungsteno a taglio incrociato, carta smerigliata e lucidatura con gommino, feltrino e pasta lucidante; per quelle in silicone si eliminavano le sbavature con un oggetto tagliente, si utilizzava il disco "lisko", presente nella confezione, per eliminare le ultime imperfezioni e per la rifinitura delle superfici rifilate è stato utilizza- 
to il glazing base-catalizzatore rendendo le pastiglie-campione lisce.

\section{RISULTATI}

S. aureus (tabella1) ha evidenziato una significativa adesività solo in presenza di saliva e su materiale ruvido $(\mathrm{p}=0.05) . S$. oralis ha rivelato qualità adesive su materiale lucido ( $\mathrm{p}=0.094)$ e ruvido $(p=0.011)$ mentre la presenza di saliva ha influenzato il numero delle cellule adese su entrambi i preparati, $p=0.33$ e $p=0.4$ rispettivamente lucido e ruvido.

C. albicans ha manifestato proprietà adesive solo sul materiale lucido $(\mathrm{p}=0.089)$ in tutte le altre condizioni sperimentali la quota di cellule adese è apparsa trascurabile.

\section{DISCUSSIONE}

Tenuto conto dei risultati ottenuti e tenuto conto del fatto che le condizioni del cavo orale, data la grande variabilità, sono difficili da riprodurre sperimentalmente, si può affermare che la metodica di lucidatura dei materiali protesici non ha influito nell'adesione batterica; mentre si può affermare che la presenza della pellicola salivare ha ridotto la capacità di aderire dei microrganismi sui materiali saggiati.

Va altresì considerato il fatto che i preparati mostrano diverse caratteristiche nei confronti dei patogeni saggiati, indice questo di un possibile ruolo dei materiali sulla qualità delle specie che possono aderire.

I materiali dentari potrebbero essere estremamente utili, come modello in vitro, nell'esplorare la capacità e i determinati dell'involucro dei microorganismi di aderire non solo ai substrati inerti ma anche ai denti.

Tabella I. Adesività dei microrganismi studiati ai materiali Dentari

\begin{tabular}{|c|c|c|c|}
\hline Materiale & Ceppo & Adesività & $\mathbf{p}$ \\
\hline \multicolumn{4}{|l|}{ Lucido } \\
\hline & S. aureus & $1.27 \pm 1.6 \times 10^{9}$ & 0.3 \\
\hline & S. oralis & $1.21 \pm 1.1 \times 10^{9}$ & 0.094 \\
\hline & C. albicans & $3.6 \pm 1.1 \times 10^{8}$ & 0.089 \\
\hline \multicolumn{4}{|l|}{ Con saliva } \\
\hline & S. aureus & $3.45 \pm 2.3 \times 10^{8}$ & 0.15 \\
\hline & S. oralis & $4.80 \pm 1.9 \times 10^{8}$ & 0.33 \\
\hline & C. albicans & $1.21 \pm 1.7 \times 10^{9}$ & 0.4 \\
\hline \multicolumn{4}{|l|}{ Ruvido } \\
\hline & S. aureus & $1.28 \pm 1.6 \times 10^{9}$ & 0.17 \\
\hline & S. oralis & $1.23 \pm 1.5 \times 10^{9}$ & 0.011 \\
\hline & C. albicans & $5.40 \pm 0.9 \times 10^{8}$ & 0.26 \\
\hline \multicolumn{4}{|l|}{ Con saliva } \\
\hline & S. aureus & $4.47 \pm 2.3 \times 10^{8}$ & 0.05 \\
\hline & S. oralis & $5.20 \pm 3.4 \times 10^{8}$ & 0.4 \\
\hline & C. albicans & $4.20 \pm 2.8 \times 10^{8}$ & 0.31 \\
\hline
\end{tabular}

\section{BIBLIOGRAFIA}

1. Bollen CML, Lambrechts $\mathrm{P}$, Quirynen $\mathrm{M}$. Comparison of surface roughness of oral hard materials to the threshold surface roughness for bacterial plaque retention. A review of the literature. Dent Mater 1997; 13: 258-69.

2. Kagermeier-Callaway AS, Willershausen B, Frank T, Stender E. In vireo colonization of acrylic resin denture base materials by Strepococcus oralis and Actinomyces viscosus. Int Dent J; 50: 79-85.

3. Khasawneh S, al-Wahadni A. Control of denture plaque and mucosal inflammation in denture wearers. Ir Dent Assoc 2002; 48: 132-8.

4. Kulak Y, Arikan A, Kazazoglu E. Existence of Candida albicans and microorganisms in denture stomatitis patiens. J Oral Rehabil 1997; 24: 788-90.

5. Kulak-Ozkan Y, Kazazoglu E, Arikan A. Oral hygiene habits, denture cleanliness, presence of yeasts and stomatitis in elderly people. J Oral Rehabil 2002; 29: 300-4.

6. Mojon P, Rentsch A, Budtz-Jorgensen E. Relationship between prosthodontic status, caries, and periodontal disease in a geriatric population. Int $\mathrm{J}$ Prosthodont 1995; 8: 564-71.

7. Morgan TD, Wilson M. The effects of surface roughness and type of denture acrylic on biofilm formation by Streptococcus oralis in a constant depth film fermentor. J Applied Microbiol 2001; 91: 47-53.

8. Nair RJ, Samaranayake LP. The effect of oral commensal bacteria on candidal adhesion to denture acrylic surfaces. APMIS 1996; 104: 339-49.

9. Petridis H, Hempton TJ. Periodontal considerations in removable partial denture treatment: a review of the literature. Int J Prosthodont 2001; 14: 164-72.

10. Quirynen M, Bollen CML. The influence of surface roughness and free surface energy on supra- and subgingival claque formation in man. A review of the literature. J Clin Periodontol 1995; 22: 1-14.

11. Quirynen M, Marechal M, Busscher HJ, Weerkamp AH, Darius PL, Van Steenberghe D. The influence of surface free energy and surface roughness on early claque formation. J Clin Periodontol 1990; 17: 13844.

12. Radford DR, Challacombe SJ, Walter JD. Denture plaque and adherence of Candida albicans to denturebase materials in vivo and in vitro. Crit Rev Oral Biol Med 1999; 10: 99-116.

13. Radford DR, Sweet SP, Challacombe SJ, Walter JD. Adherence of Candida albicans to denture-base materials with different surface finishes. J Dent 1998; 26 : 577-83.

14. Radford DR, Watson TF, Walter JD, Challacombe SJ. The effects of surface machining on heat acrylic resin and two soft denture base materials: a scanning electron microscope and conofocal microscope evaluation. J Prosthet Dent 1997; 77: 200-8.

15. Taylor R, Maryan C, Verran J. Retention of oral microorganisms on cobalt-chromium alloy and dental acrylic resin with different surface finishes. J Prosthet Dent 1998; 80: 592-7.

16. Verran J, Maryan CJ. Retention of Candida albicans on acrylic resin and silicone of different surface topography. J Prosthet Dent 1997; 77: 535-9. 
17. Yeung AL, Lo EC, Chow TW, Clark RK. Oral health status of patients 5-6 years after placement of cobaltchromium removable partial dentures. J Oral Rehabil 2000; 27: 183-9.

18. Zlararic DK, Celebic A, Valentic-Peruzovic M. The effect of removable partial dentures on periodontal health of abutement and non-abutement teeth. J Periodontol 2002; 73: 137-44.

Eugenio A. Debbia

Sezione di Microbiologia, DISCAT Università di Genova

Largo R. Benzi 10 - 16132 Genova

Tel. 0103537655 ,

E-mail: debbia@unige.it 